\title{
SOlar Neutron TRACking (SONTRAC) Concept
}

\author{
G.A. de Nolfo*1, A. Bruno ${ }^{1}$, J. Dumonthier ${ }^{1}$, I. Liceaga-Indart ${ }^{1,2}$, J. Legere $^{3}$, R. \\ Messner $^{4}$, J. G. Mitchell ${ }^{1,5}$, J.M. Ryan ${ }^{3}$, G. Suarez ${ }^{1}$, T. Tatoli ${ }^{1,6}$ \\ ${ }^{1}$ NASA Goddard Space Flight Center, Greenbelt, MD \\ E-mail: georgia.a.denolfo@nasa.gov \\ ${ }^{2}$ Catholic University, Washington D.C. \\ ${ }^{3}$ Space Science Center, University of New Hampshire, Durham, NH \\ ${ }^{4}$ ECE Department, College of Engineering and Physical Sciences, University of New Hampshire, \\ Durham, $\mathrm{NH}$ \\ ${ }^{5}$ George Washington University, Department of Physics, Washington D.C. \\ ${ }^{6}$ University of Maryland, Baltimore County, MD
}

\begin{abstract}
The detection of fast neutrons has important applications in a variety of fields including geospace, solar, and planetary physics. Though neutrons are ubiquitous products of nuclear interactions, they are challenging to detect and the measurements typically suffer large backgrounds. Highenergy neutrons (> $50 \mathrm{MeV}$ ) pose even greater challenges because the traditional double scatter technique based on a time-of-flight (ToF) is limited by the finite flight path and active detector sizes limited by small satellite platforms. At these high energies, the proton recoil is likely to escape the detector volume, degrading the energy and angular resolution. Scintillator-based technologies have a proven record for detecting and measuring fast neutrons. They have high stopping power, good energy resolution, and fast timing properties. By dramatically increasing the segmentation of scintillator arrays (down to hundreds of sub-mm fibers) proton-tracking can be achieved, effectively supplanting the ToF measurement, thereby eliminating the need for widely separated detectors, thereby greatly increasing the detection efficiency. It reduces the scale size of the detector from that necessary for time of flight to the proton range in dense matter. Modern readout devices such as silicon photomultipliers offer an ideal alternative to photomultiplier tubes given their inherently compact size, fast response, and low operating voltages. The Solar Neutron TRACking (SONTRAC) Concept, based on scintillating-fiber bundles, would provide high-resolution imaging of fast neutrons at energies where the bulk of solar and magnetospheric neutrons resides. Recent development and performance of the SONTRAC Concept are presented.
\end{abstract}

36th International Cosmic Ray Conference -ICRC2019-

July 24th - August 1st, 2019

Madison, WI, U.S.A.

${ }^{*}$ Speaker. 


\section{Introduction}

Solar flares can be prodigious producers of energetic particles, and are thus rich laboratories for studying particle acceleration physics. However, few of the particles accelerated in flares reach spacecraft to be measured. Instead, we typically know of these particles through the secondary neutral radiation they emit when they interact with the solar atmosphere. This radiation takes various forms in the $\gamma$ channel, such as lines, pion-related emission and bremsstrahlung. Neutrons are also produced and are most readily seen when they combine with hydrogen producing deuterium and a $2.223 \mathrm{MeV} \gamma$ ray. Direct neutrons have been detected in several flares when the intensity is great enough, but typically with little or no spectroscopic information (Chupp et al. 1987; Murphy et al. 1999). Less than a handful of flares, however, produced neutrons that were measured for their energy on a neutron-by-neutron basis.

Gamma-ray and neutron observations, in particular, sample the energy spectrum of solar flare protons and ions in specific and not necessarily overlapping energy ranges. Exactly how solar flare protons and ions are accelerated and to what extent these acceleration processes are energy dependent is still not well understood (Vestrand Miller 1999). If solar flare particle acceleration obeys multiple energy dependent acceleration time scales, one should witness this as different reaction channels turn on at different times and last for different amounts of time depending on the plasma processes and acceleration mechanisms at play. If only nuclear lines are detected, the sampled energy range may be too small to see this dispersive effect. Therefore, being able to measure neutrons and pion-associated $\gamma$ rays is important for investigating this process, if it is present.

Neutrons are also produced in the same reactions. High energy neutrons are predominantly produced via $\mathrm{p}-\alpha$ and $\alpha-\alpha$ interactions. In addition, neutrons can be produced from the interactions of proton and helium with carbon and heavier ions (Hua Lingenfelter 1987; Murphy et al. 2007). Weighting neutron cross sections with the photospheric abundances and the energetic particle composition derived from the 1980 \& 1982 flares (Murphy, Dermer, \& Ramaty 1987), the neutron production can be classified into four separate energy regimes (Nieminen 1997). Neutrons are dominated by 1) the $\alpha$-CNO interactions with energies $<10 \mathrm{MeV}, 2)$ the $\mathrm{p}-\mathrm{CNO}$ and $\alpha$ - $\alpha$ interactions with energies $\sim 10-100 \mathrm{MeV}, 3$ ) the p- $\alpha$ interactions with energies $100-500 \mathrm{MeV}$, and 4) the p-p interaction above $500 \mathrm{MeV}$. Using production ratios, Chupp (1988) derived the hydrogen density of $\sim 10^{12} \mathrm{~cm}^{-3}$, typical chromospheric densities, necessary for the production of neutrons, suggesting that neutrons are generated deep in the chromosphere and photosphere (Hua Lingenfelter 1987).

The combination of $\gamma$-ray and neutron time-dependent spectra provides a strong constraint on the spectral shape and the temporal evolution of flare particles, since the production cross sections are quite different and depend on the accelerated proton/ion energy (Lockwood, Debrunner, \& Ryan 1997). The ratio of the nuclear $\gamma$-ray lines at 4-7 MeV band and $2.223 \mathrm{MeV}$, which strongly depends on the accelerated ions at the flare, provides a measure of the accelerated ion spectrum and number (Mandzhavidze Ramaty 1993). The $\gamma$-ray production from pion decays begins at a threshold energy $\sim 300 \mathrm{MeV} /$ nucleon, reaches a peak, and essentially follows the spectral shape of the accelerated ions at high energies. The neutron production also essentially follows the accelerated ion powerlaw behavior at high energies but more importantly covers a very broad range in ion energy from 
$50-300 \mathrm{MeV} /$ nucleon. It is clear that neutrons bridge an important gap in ion energies at the solar flare.

A good illustration of the importance in using $\gamma$-ray and neutron observations to constrain the accelerated ion spectrum at the flare is the 1982 June 3 flare. GRS/SMM detected pion-related emission during the impulsive phase that indicated ion acceleration to $\mathrm{GeV}$ energies within $10 \mathrm{~s}$ (Chupp, et al. 1987) but also emission lasting for $\sim 20$ minutes afterwards (Forrest et al. 1985). High-energy neutrons ( $>50 \mathrm{MeV}$ ) were also measured for the 1982 June 3 flare with GRS (Chupp et al. 1985) in addition to an indirect detection with ground level neutron monitors (Debrunner et al. 1983; Debrunner, Fluckiger, Lockwood 1990). The detection of pion-related emission and $\mathrm{GeV}$ neutrons well after the impulsive phase introduces an important high-energy sample of the accelerated proton spectrum implying a hard proton spectrum that emerges on a different time scale than the usual nuclear-line emission.

Solar flare neutrons were first detected in space with the GRS on 1980 June 21 (Forrest, et al. 1980) and have been identified by Chupp et al. (1984), Debrunner et al. (1983) and Dunphy et al. (1992), although the spectroscopy of these observations are generally limited. With no neutron spectral information one must assume a production profile and interpret the rate of neutron detection accordingly, eliminating virtually any possibility of investigating a time dependent proton/ion spectrum within the flare. Such modeling was conducted by Debrunner et al. (1990)for the 1982 June 3 flare and by Watanabe et al. (2006) for several flares. The neutrons were detected however, at ground level and have energies comparable to the protons and ions responsible for pion production. Thus, the production of pion-associated $\gamma$ rays should accurately follow the production of Ground Level events (GLE) neutrons, thus simplifying the modeling exercise if one measures pion-related $\gamma$ emission, such as with Fermi/LAT (Atwood et al. 2007).

The spectral shape and composition of solar flare energetic particles have been determined almost exclusively by $\gamma$-ray observations. Solar neutron observations are rare and have been relegated primarily to non-spectroscopic observations. However, COMPTEL/CGRO offered the first clear distinction between solar neutrons and photons and perhaps more importantly measure the neutron energy on a neutron-by-neutron basis. Ryan et al. (1992) measured solar neutrons from the 1991 Jun 9 flare with an energy resolution of $\sim 20 \%$, which corresponds to an uncertainty in the neutron release time of about a minute (Ryan et al. 1992).

A solar neutron measurement from $20-200 \mathrm{MeV}$ is important when combined with high- and low-energy $\gamma$-ray measurements. In this range, neutron survival to $1 \mathrm{AU}$ is likely, and is coincidentally the best range to fully sample a wide swath of the energetic ion population. Furthermore, the dominant source of the inner radiation belt protons near $100 \mathrm{MeV}$ arises from the decay of secondary neutrons produced in the Earth's atmosphere, e.g., CRAND (Singer 1958; Jentsch 1981; Selesnick et al. 2007). Similar physics to the CRAND physics at Earth takes place at other planets with magnetospheres, i.e., Jupiter and Saturn (Kollmann et al. 2013). Validating the CRAND model at Earth would enhance the science return of future planetary missions.

In addition to the above applications, improved knowledge of atmospheric neutrons themselves, including spatial and temporal variations, are valuable because neutron-induced single event upsets in avionics systems constitute a reliability problem (Leray 2007). Furthermore, neutrons are penetrating, dangerous and abundant, posing a health and safety risk to astronauts and aircraft personnel (Dyer 2002). Neutrons also constitute a major background for other NASA assets in LEO 
(e.g., Wunderer et al. 2006; Ormes et al. 2007).

\section{SOLAR NEUTRON TRACKING CONCEPT}

The neutron/ $\gamma$-ray spectrometer, SONTRAC, first conceptually introduced by Glenn Frye et al. (1985), provides a means to measure neutrons in the range of 20-150 MeV in a compact envelope with high efficiency, ideal for a small spacecraft and/or deep-space probes. The SONTRAC concept relies on the measurement of the momentum vector of the recoil proton associated with two interactions within a single scattering volume, e.g., the highly segmented fiber bundle. From a measure of the recoil moment in two successive scatters, the energy and direction of the incident neutron can be readily reconstructed (Figure 1). A system that measures the parameters of both recoil proton tracks in 3-d, provides the necessary and sufficient information to determine the incident neutron energy and direction with no azimuthal ambiguity (Ryan et al. 1993, 2012). The angular and energy resolutions depend on the ability to accurately measure the proton tracks. Imaging allows for a more complete separation of the source signal from the background. By allowing the scatter to take place in a single large block, the solid-angle factor between the scatters is much greater than that for widely separated detectors utilizing the time-of-flight technique, increasing the efficiency. As such, it would have wide fields of view, front and back.

SONTRAC was originally developed for the study of high-energy solar flare processes, but it was expanded to atmospheric physics, radiation therapy, and nuclear materials monitoring (Bravar et al. 2005). Because the original SONTRAC was based on scintillating fibers readout by image intensifiers with CCD detectors, the concept exhibited limitations including slow event rate, large data volume and large physical size/mass (Ryan et al. 2003). The upgraded SONTRAC (discussed in this paper) consists of orthogonally stacked, alternating layers of parallel scintillating plastic fibers read out by arrays of silicon photomultipliers (SiPMs), see Figure 1. The prototype consists of a $35 \times 35$ scintillating fiber bundle with a $1.36 \mathrm{~mm}$ fiber pitch (Fig. ??). Each of the fiber bundle sides is paired with a $32 \times 32$ array of 1-mm SiPMs by KETEK (inset to Fig. ??) with the

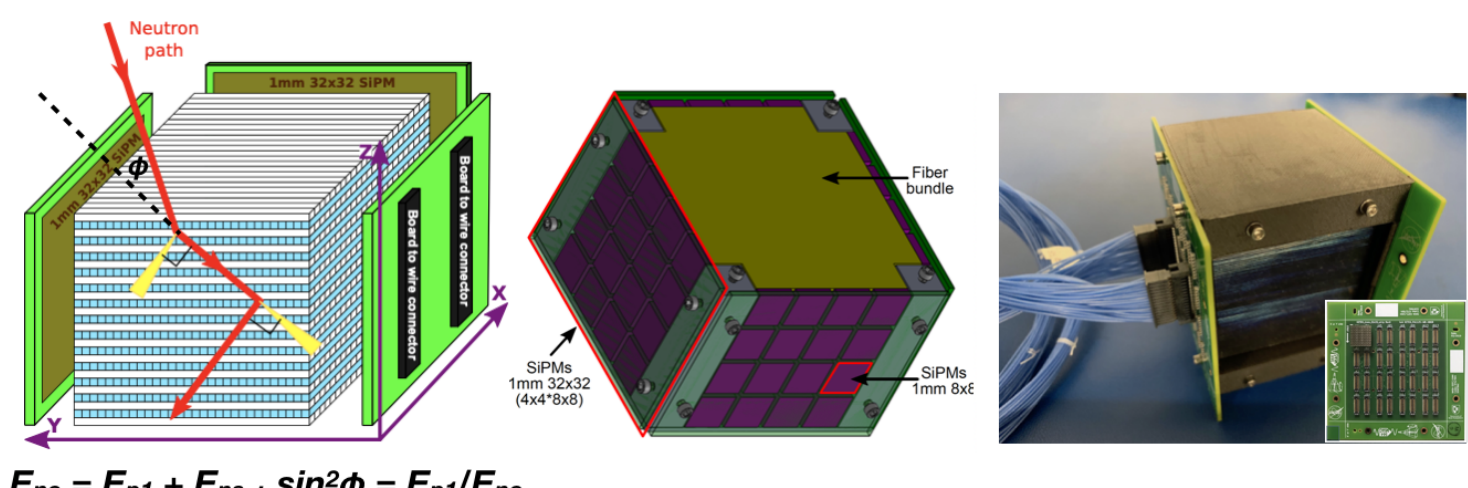

Figure 1: (left) SONTRAC instrument consisting of orthogonally stacked plastic scintillators readout by arrays of SiPMs. A measurement of two recoil tracks from fast neutron interactions determines the incident neutron energy and direction. (right) Mechanical housing for SONTRAC showing the tiling of sixteen $8 \times 8$ 1-mm SiPM arrays on four surfaces. 
same pitch as the fibers, providing a stereoscopic view for proton tracking. SiPMs, with $<1$-ns rise times, good quantum efficiency, and low noise, offer a compact, robust, low-power readout. Commercial arrays of 1-mm SiPMs with a pitch of $1.36 \mathrm{~mm}$ are readily available (e.g., KETEK), however, custom SiPMs can be manufactured with a pitch down to several-hundred $\mu \mathrm{m}$, enabling a future configuration with significantly lower energy threshold detection.

A SiPM-based neutron imaging spectrometer is a significant improvement over earlier protontracking based technologies (Ryan et al. 1999; Miller et al. 2005; Legere et al. 2006; Muraki et al. 2012; 2013). Yamaoka et al. recently proposed a CubeSat Neutron Sensor (CNS) using SiPM readout coupled to a fiber block. SONTRAC offers better spatial resolution (1.36-mm as apposed to 4-mm) and much greater data compression based on our novel strip readout technique (see discussion below).

Individual SiPMs can easily trigger on the several photoelectrons expected from a minimum ionizing particle (MIP) in 1-mm fibers. The large dynamic range allows the proton-recoil Bragg peak to be recognized. The 1.36-mm pitch of the current array imposes a minimum proton energy of $\sim 25 \mathrm{MeV}$ for tracking, or a double scatter neutron threshold of $\sim 50 \mathrm{MeV}$, although lower thresholds $(\sim 10 \mathrm{MeV})$ are possible with smaller custom SiPMs.

SONTRAC's finely grained SiPM array is read out in three 1-d anode strips rather than in the form of numerous individual anodes, significantly reducing the number of channels to process, i.e., several 1-d projections, as opposed to 2-d stereoscopic images. This reduces the total number of channels from 2048 ( 2 sides, 1024 per side) to 128 (4 sides, 32 per side). For a small satellite (e.g. CubeSat), 128 channels is still a large number to be read-out with discrete electronics. Highly integrated ASICs are a better option. Signal readout for the prototype SONTRAC utilizes the Petiroc2A (https://www.caen.it/products/dt5550w/), a 32-channel readout ASIC to process the incoming signal from the each of the SiPM detector strips. The Petiroc2A ASIC reduces the size, mass and power while still processing a large number of channels at a high rate. The prototype relies on the Petiroc2A readout system from CAEN (CAEN 2019) which consists of a field programmable gate array (FPGA) that can be used to test the hardware-based track recognition algorithms in combination with the strip readout.

\section{PERFORMANCE}

The SONTRAC prototype has been integrated and is currently undergoing tests using ground level muons and radioactive sources, including a DT generator and Cf-242. Evaluation of the ionization energy loss along the recoil proton tracks will be compared with GEANT4 simulations in order to properly identify the Bragg peaks. Track reconstruction using ground-level muons will be used to evaluate the energy and angular response performance. Accelerator tests are planned for 2020. Figure 2(a) shows the SiPM response to a ground-level muon passing through four vertically stacked scintillating fibers in the bundle and readout by individual 1-mm SiPMs, demonstrating sensitivity to minimum ionizing particles.

Track reconstruction takes advantage of the 1-d projections and pulse height information along the track, as an example see the simulated 2-d tracks in Figure 2(b). The increased energy deposit along the track due to the well-known Bragg peak provides track orientation information (e.g., upward versus downward going tracks). 

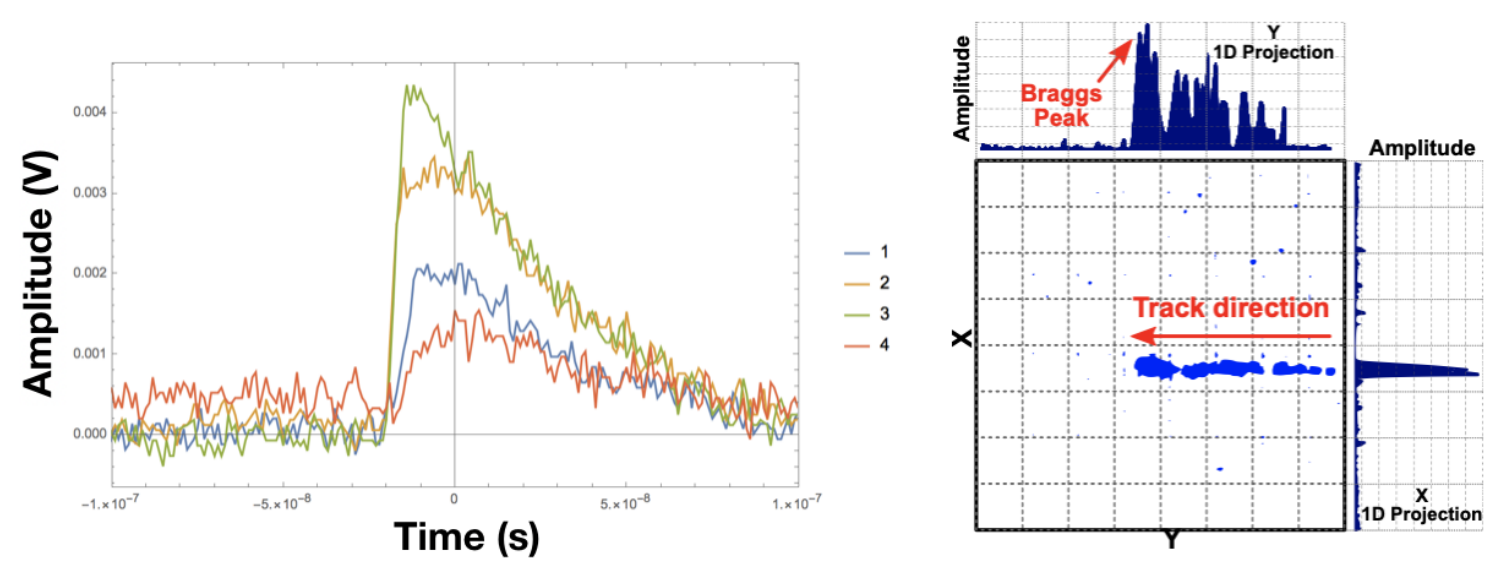

Figure 2: (a) Signals from ground-level muons within individual fibers in SONTRAC. (b) Simulated track reconstruction demonstrating the utility of the Bragg peak.

Simulations of SONTRAC have been conducted using the CERN software package GEANT4. Figure 3 shows a simulated neutron track and resulting recoil proton tracks for an incident neutron of energy $115.95 \mathrm{MeV}$. The proton recoil tracks encompass numerous fibers, extending a total of $>$ $20 \mathrm{~mm}$ in total length. Figure 4(a) shows the more general case for incident neutrons with energy $91 \mathrm{MeV}$ in which multiple fibers often register the recoil proton track, providing numerous opportunities to measure the proton recoil track adequately and thus properly reconstruct the incident neutron energy and direction. The detection efficiency for registering two proton recoils within the SONTRAC fiber bundle volume of $107 \mathrm{~cm}^{3}$ is shown in Figure 4 (b) where a selection has been applied for tracks that include at least 2 hit fibers.

\section{SUMMARY}

Preliminary tests of the SONTRAC prototype show good sensitivity to minimum ionizing tracks based on ground-level muons. The threshold energy, sensitivity to the Bragg peak, and angular resolution will be explored in detail with an accelerator run in the spring of 2020.

We envision an instrument that is scalable to fit in either a 3U/6U or $12 \mathrm{U}$ CubeSat platform, depending on the science requirements, e.g. a $(10-\mathrm{cm})^{3}$ neutron tracker, surrounded by charged particle anti-coincidence detector. Such a proposed instrument would have an effective area for detecting $30-80 \mathrm{MeV}$ neutrons of $2 \mathrm{~cm}^{2}$, approximately the neutron effective area of the 1.5ton imaging Compton telescope, COMPTEL. A CubeSat instrument, because of its small mass, would be far more sensitive due to the small spacecraft background. We estimate the typical energy resolution of $10 \%$ or better for the majority of neutron events. The angular resolution is determined by the pitch of the fibers, which translates to the uncertainty in the end points of the particle tracks. For a $45^{\circ}$ scatter and 300- $\mu \mathrm{m}$ fiber pitch (possible with smaller sized arrays of SiPMs), it ranges from $23^{\circ}$ at $20 \mathrm{MeV}$ to $5^{\circ}$ at $50 \mathrm{MeV}$ to $0.7^{\circ}$ at $200 \mathrm{MeV}$. This good angular resolution yields a high signal to noise and thus good sensitivity. Larger versions of the fiber-based neutron spectrometer 

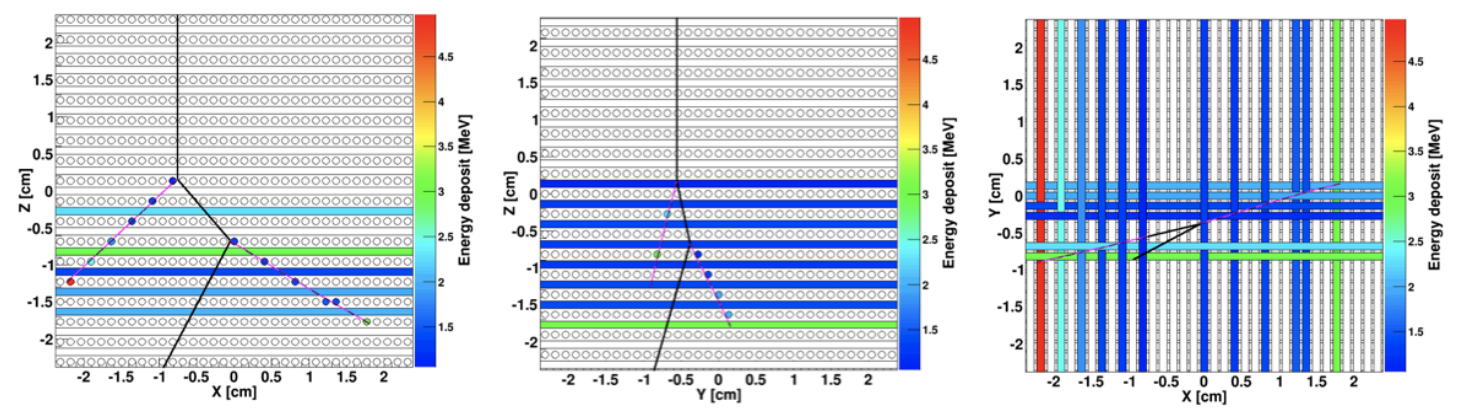

Figure 3: Simulated $115.95 \mathrm{MeV}$ neutron track (black) showing two interactions and the resulting proton recoil tracks (colored circles). The first and second proton recoil track are 20 and $22 \mathrm{~mm}$ in length. (a)Z versus $\mathrm{X}$ projection, (b) $\mathrm{Z}$ versus $\mathrm{Y}$ projection, and (c) X versus $\mathrm{Y}$ projection.
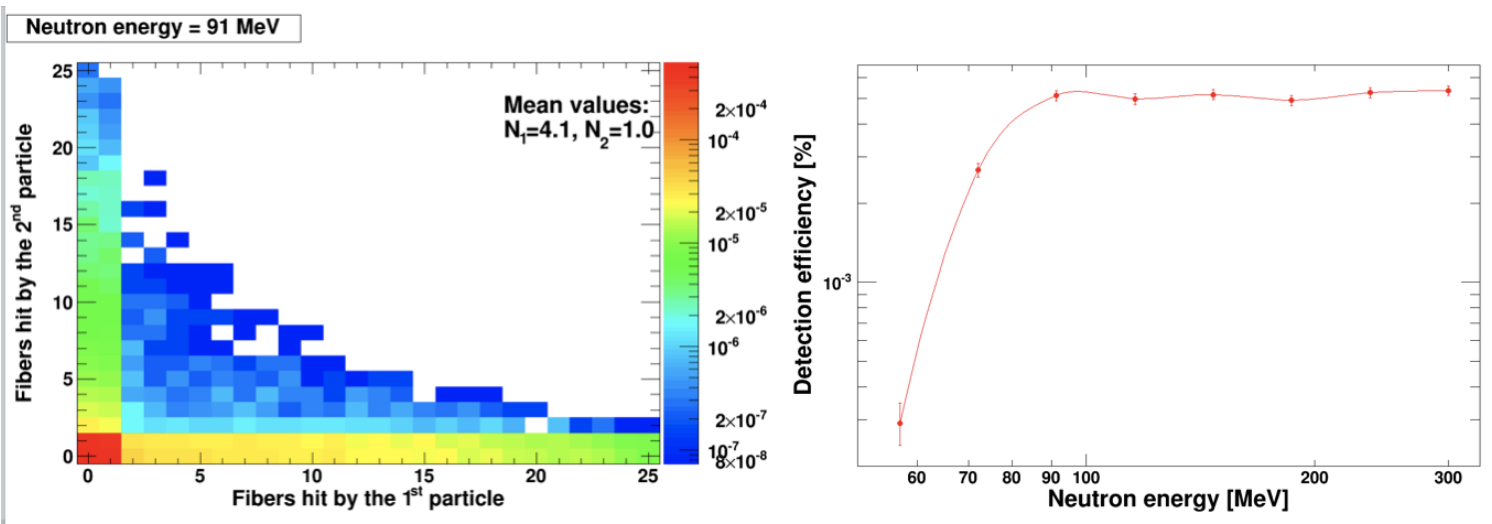

Figure 4: (a) Number of fibers registering a proton recoil track for double scatter neutrons with neutron energy $91 \mathrm{MeV}$. (b) Detection efficiency for a double scatter neutron creating two recoil proton tracks in the fiber bundle, where a minimum 2 fiber selection has been applied.

are possible such that an entire $4 \mathrm{U}$ could be dedicated to scintillating fibers and readout (perhaps by assembling small blocks), significantly improving the effective area.

\section{References}

[1] E.L., Chupp, et al., 1987, ApJ, vol. 318, 913

[2] W.B., Atwood, et al., 2009, ApJ, vol. 697, 1071

[3] E.L., Chupp, et al., 1988, Solar Physics, vol. 118, 137

[4] E.L., Chupp, et al., 1984, Annual Review of Astronomy and Astrophysics, 22, 359

[5] E.L., Chupp, et al., 1985, 19th ICRC, 126

[6] H., Debrunner, et al., 1983, 18th ICRC, 75

[7] H., Debrunner, et al., 1990, ApJ, vol 73, 259

[8] P.P., Dunphy, et al., 1992, in Proc. of the Particle Acceleration in Cosmic Plasmas Workshop, eds. G. P. Zank, \& T. K. Gaisser (Newark, DE: AIP:New York), 253 
[9] R.J., Murphy, et al., 1999, ApJ, vol. 510, 1011

[10] K., Watanabe, et al., 2006, ApJ, vol. 636, 10

[11] D.J., Forest, et al., 1985, 19th ICRC, 146

[12] W.T., Vestrand, et al., 1999, in the Many Faces of the Sun, eds. K.T. Strong, J.L.R. Saba, B.M. Haisch, and J.T. Schmelz (New York: Springer-Verlag), 231

[13] X.M., Hua, et al., 1987, Solar Physics, vol. 107, 351

[14] R.J., Murphy, et al., 2007, ApJ Suppl, vol. 168, 38

[15] R.J., Murphy, et al., 1987, ApJ Suppl, vol. 63, 721

[16] P., Nieminen, et al., 1997, Thesis, 137

[17] J.A., Lockwood, et al., 1997, Solar Physics, vol. 173, 151

[18] N., Mandzhavidze, et al., 1993, Nuclear Physics B, vol. 33, 141

[19] R.E., Lingenfelter, et al., 1965, JGR., vol. 70, 4087

[20] J.M., Ryan, et al.,2000, Space Sci. Rev., 93, 581

[21] S. F., Singer, 1958, Phys. Rev. Lett., 1, 181

[22] J.M., Ryan, 1992, in Data Analysis in Astronomy, COMPTEL as a Solar Gamma Ray and Neutron Detector, ed. V. D. GesÃž (Plenum Press: New York), 261

[23] R. S., Selesnick, et al., 2007, Space Weather, 5

[24] U., Bravar, 2005, IEEE, 2, 634

[25] V., Jentsch, 1981, J. Geophys. Res., 86, 701

[26] J. L., Leray, 2007, Microelectronics Reliability, 47, 1827

[27] P., Kollman, 2013, Icarus, 222, 323

[28] C., Dyer, 2002, Eur. Space Agency Spec. Publ., ESA SP-477, 505

[29] J. F., Ormes, et al., 2007, AIP Conf. Proc. 921, 560

[30] G., Suarez, et al., 2019, IEEE, this proceedings.

[31] C. B., Wunderer, et al. 2006, New Astronomy Reviews, 50, 608

[32] G.M., Frye, et al., 19th ICRC, 5, 498, 1985

[33] J.M., Ryan, et al., 2003, SPIE, 4853, 399

[34] J. M., Ryan, et al., 2012, Proc. SPIE, 8509

[35] J. M., Ryan, et al. 1999, IEEE NSS Conference Record, 483

[36] R.S., Miller,et al., 2003, NIM-A, 505, 36

[37] Y., Muraki, et al., 2012, Adv. in Astronomy; doi:10.1155/2012/379304

[38] Y., Muraki, et al., 2013, Proc. 33rd ICRC; arXiv:1307.5376

[39] J. S., Legere, et al., 2006 IEEE NSS Conference Record, 417

[40] T., Yamaoka, et al., 2018 Int. Soc. for Optics and Phot., p.107620J 